\title{
Preparation of PEGylated Liposomal Ginsenoside; Formulation Design and in vitro Evaluation
}

\author{
Y. Q. CUI, P. YANG ${ }^{1}$, P. SUN ${ }^{2}$, Y.D.YAN ${ }^{3}$, G.Y.JIN ${ }^{4 *}$ AND J.S.QUAN* \\ Department of pharmaceutics, College of pharmacy, Yanbian University, ${ }^{1}$ Antu County Health Supervision Institute, \\ ${ }^{2}$ Department of pharmacy, Yanbian University Hospital, Jilin, 133000, 3BBeijing Fogangren Bio-Pharma Limited, Beijing, \\ 102628, ${ }^{4}$ Department of Radiology, Yanbian University Hospital, Jilin, 133000, China
}

Cui et al.: Ginsenoside Rg3-loaded PEGylated liposomes

\begin{abstract}
In this study, ginsenoside Rg3-loaded PEGylated liposomes were prepared and optimized using the BoxBehnken design. These liposomes were characterized, the cumulative release profiles were investigated and compared with ginsenoside $\mathrm{Rg}_{3}$-loaded liposomes in vitro. To improve the stability ginsenoside Rg3-loaded PEGylated liposomes were freeze-dried and the lyoprotectants to be added were screened. The results showed that the liposomes have a small particle size $(152.58 \pm 0.74 \mathrm{~nm})$ and spherical shape. The encapsulation efficiency and drug-loading rate were approximately $85.24 \pm 1.02$ and $7.44 \pm 0.08 \%$, respectively. For lyoprotectants, 2 \% lactose was chosen as the lyophilized protectant according to the appearance, re-dispersity, particle size, and entrapment efficiency of lyophilization of ginsenoside Rg3loaded PEGylated liposomes. In vitro release showed that ginsenoside Rg3-loaded PEGylated liposomes showed a more obvious sustained release effect, which suggests that ginsenoside Rg3-loaded PEGylated liposomes might enhance the therapeutic effect of ginsenoside Rg3.
\end{abstract}

Key words: Ginsenoside Rg3; PEGylated liposomes; Box-Behnken design

Ginseng, the root of Panax ginseng, has been used globally for thousands of years as a herbal drug ${ }^{[1]}$. Ginsenoside Rg3 (G-Rg3), a primary bioactive component of $P$. ginseng, has been extracted and studied extensively for its anticancer properties. Ginseng can prevent tumor cell adhesion, invasion and metastasis. Furthermore, it can inhibit tumor cell proliferation and induce apoptosis ${ }^{[2]}$. The combination of G-Rg3 with other chemotherapeutics has been found to have a synergistic effect ${ }^{[3-5]}$. Although G-Rg3 possesses a variety of medical activities, the clinical application of G-Rg3 is restricted due to its poor water solubility, short circulation half-life and poor target specificity ${ }^{[2,6,7]}$. For this reason, to improve the bioavailability and therapeutic effect of G-Rg3, current research is focused on improving its delivery efficiency by developing new formulations. Zhang et al. ${ }^{[8]}$ prepared a G-Rg3 inclusion compound using a magnetic stirring method. Compared to G-Rg3, the solubility of the inclusion compound was enhanced approximately 19 fold. Geng et al. ${ }^{[9]}$ prepared G-Rg3-loaded PEG/PLGA nanoparticles. Compared to G-Rg3, G-Rg3-loaded nanoparticles had a

*Address for correspondence

E-mail: jishanquan@sina.com

January-February 2020 sustained-release effect and maintained a suitable blood concentration over a long period of time. In addition, G-Rg3-loaded PEG/PLGA nanoparticles promoted the antitumor effect of G-Rg3 in Lewis lung cancer mice. Yu et al. ${ }^{[6]}$ found that the cytotoxicity and tumor inhibitory potential of liposomal G-Rg3 was significantly higher than the G-Rg3 solution group.

In recent years, stable circulating liposomes have been studied as they can improve the in vivo half-lives of conventional liposomes by reducing macrophage phagocytosis to reduce the clearance ${ }^{[10-14]}$. Through the enhanced permeability and retention (EPR) effects on solid tumors, long-circulating liposomes can also increase the concentration of a drug at the target area, thereby achieving a better anticancer therapeutic effect. Studies have also found that PEGylated liposomes

This is an open access article distributed under the terms of the Creative Commons Attribution-NonCommercial-ShareAlike 3.0 License, which allows others to remix, tweak, and build upon the work non-commercially, as long as the author is credited and the new creations are licensed under the identical terms

Accepted 02 December 2019

Revised 18 September 2019

Received 28 Juy 2019

Indian J Pharm Sci 2020;82(1):149-156 
are stable for up to several months of storage at low temperature ${ }^{[15]}$. In this study, PEGylated liposomes were prepared to deliver G-Rg3 and their potential investigated to provide a direction for future anticancer research.

A reasonable experimental design is very important, especially when complex formulations need to be developed, as it can save time, money and reduce experimental errors to obtain reliable experimental data. In particular, the multivariate strategy of experimental design allows simultaneous investigation of the effects of several variables, their actual significance on the considered response and the possible interrelationship among them. This approach yields maximum information with a small number of experiments ${ }^{[16]}$. Response surface methodology (RSM) explores the relationships between several independent variables and one or more response variables ${ }^{[17]}$. RSM includes central composite design (CCD), Box-Behnken design (BBD) and Doehlert design (DM). Present study used BBD since it has a high fitting correlation coefficient, good predictability and high precision and has been considered to be a cost-effective technique compared to the other usual processes of formulation and optimization because it requires fewer experimental runs and therefore saves time ${ }^{[18]}$.

In this study, the formulation process of G-Rg3-loaded PEGylated liposomes (G-Rg3-PLP) were screened using a three-level three-factor BBD. The independent variables selected were phosphatidylcholine:cholesterol (PC:Chol, wt/wt) ratio $\left(\mathrm{X}_{1}\right), \quad \mathrm{PC}: \mathrm{N}$-(carbonylmethoxypolyethylene glycol 2000)-1,2-distearoyl-snglycero-3-phosphoethanolamine (DSPE-PEG ${ }_{2000}$ ) ratio $(\mathrm{mol} / \mathrm{mol})\left(\mathrm{X}_{2}\right)$ and PC: $\mathrm{G}-\mathrm{Rg} 3$ ratio $(\mathrm{wt} / \mathrm{wt})\left(\mathrm{X}_{3}\right)$. The encapsulation efficiency (EE, $\mathrm{Y}_{1}$ ) and the drug-loading rate $\left(\mathrm{DL}, \mathrm{Y}_{2}\right)$ were selected as dependent variables. The characterization of the liposomes was also studied.

\section{MATERIALS AND METHODS}

G-Rg3 was purchased from Nanjing Langze Biotechnology Co., Ltd. (Nanjing, China). $\mathrm{PC}$ and N-(carbonyl-methoxypolyethylene glycol 2000)-1,2-distearoyl-sn-glycero-3phosphoethanolamine (DSPE-PEG 2000 ) were purchased from Avanti Polar Lipids Inc. (Suffolk, England). Cholesterol was purchased from Beijing Solar Bioscience \& Technology Co., Ltd. (Beijing, China). Methanol and acetonitrile were obtained from Sigma Chemical Co. (St. Louis, MO, USA). Ultrapure water used for liquid chromatography/UV/Vis spectrum (LC/
UV) was obtained using a Milli-Q water purification system.

\section{Preparation of G-Rg3-PLP:}

G-Rg3-PLP was prepared using the film dispersionultrasonic method ${ }^{[19]}$. Briefly, phosphatidylcholine, cholesterol, DSPE-PEG 2000 , and G-Rg3 were accurately weighed and dissolved in a 3:2 v/v mixture of methanol:chloroform. After these were dissolved completely, the solvent was evaporated using a rotary evaporator at $60^{\circ}$ to form a thin dry film. The film was dried further in a vacuum oven overnight. The dried lipid film was hydrated in phosphate buffered saline (PBS, $\mathrm{pH}$ 7.4) at $40^{\circ}$. The dispersion was sonicated using a Scientz-II D ultrasonic cell crusher (Zhejiang, China, $200 \mathrm{w}$, ultrasound $3 \mathrm{~s}$, intermittent $3 \mathrm{~s}$ ) to reduce particle size and then passed through a $0.45 \mu \mathrm{m}$ filter membrane to remove larger liposomes. The final products were stored at $4^{\circ}$ until further use. Blank liposomes (without G-Rg3) and conventional liposomes (without DSPE$\mathrm{PEG}_{2000}$ ) were also prepared using the same method.

\section{Characterization of G-Rg3-PLP:}

The morphology of G-Rg3-PLP was observed using a transmission electron microscope (TEM, (HT7700, Hitachi, Japan). The sample was dropped onto the grid and dried completely at room temperature. After counterstaining it with $2 \%$, w/v aqueous phosphotungstic acid solution, TEM images were taken. The particle size and zeta potential of G-Rg3-PLP were measured with a laser particle size analyser (JL-1197, Chengdu Jingxin Powder Testing Equipment Co., Ltd. Sichan, China) and zeta potential analyser (B1-90 Plus, Holtsville, New York, USA), respectively.

\section{Determination of entrapment efficiency (EE) and drug loading capacity (DL):}

One millilitre of G-Rg3-PLP solution was transferred into $1.5 \mathrm{ml}$ tubes $(\mathrm{n}=3)$ and centrifuged for $90 \mathrm{~min}$ at $15000 \mathrm{rpm}$ using a high-speed refrigerated centrifuge. The concentration of G-Rg3 $\left(\mathrm{C}_{2}\right)$ in the supernatant was analysed on a high pressure/performance liquid chromatography (HPLC) system. The precipitate was collected, dried, and weighed $\left(\mathrm{W}_{1}\right)$ and the amount of $\mathrm{G}-\mathrm{Rg} 3$ in the powder $\left(\mathrm{W}_{2}\right)$ was measured by HPLC. The same volume of G-Rg3-PLP was degraded by demulsification and the drug concentration $\left(\mathrm{C}_{1}\right)$ was measured. Data were analysed by independent-samples $t$ tests using SPSS16.0 software. $\mathrm{P}<0.05$ was considered statistically significant. The EE and DL capacity 
were calculated by the following formula: $\mathrm{EE} \%=$ $1-\mathrm{C}_{2} / \mathrm{C}_{1} \times 100$, and $\mathrm{DL} \%=\mathrm{W}_{2} / \mathrm{W}_{1} \times 100$.

\section{Box-Behnken design:}

EE is an important evaluation parameter for liposomes and is affected by the amount of PC, Chol, and DSPE$\mathrm{PEG}_{2000}$ in the liposomes ${ }^{[20-24]}$. Therefore, in this study, a three-level three-factor BBD was employed to optimize the formulation variables. Based on the preliminary experimental results, three independent variables, PC:Chol ratio (wt/wt) (X1), PC:DSPE-PEG2000 ratio (mol/mol) (X2), and PC:G-Rg3 ratio (wt/wt) (X3) were evaluated and the EE (Y1) and DL (Y2) were selected as dependent variables. In the multi-index test, considering the interplay between various factors, a comprehensive evaluation index-overall desirability (OD) was introduced and calculated by the following Eqn., $d i=(Y i-Y \min ) /(Y \max -Y \min ) \quad O D=(\mathrm{d} 1 \mathrm{~d} 2 \mathrm{~d} 3 \ldots \ldots$ $\mathrm{dn}) 1 / \mathrm{n}$. Yi is the measured value and Ymin is each index minimum value. Ymax is the index maximum value. According to experimental purposes and the standards of Chinese Pharmacopoeia, we set $\mathrm{Y} 1=70-100 \%$, $\mathrm{Y} 2=3-8 \%, \mathrm{Yi} \geq \mathrm{Ymax}, \mathrm{di}=1, \mathrm{Yi} \leq \mathrm{Ymin}$, and $\mathrm{di}=0$.

\section{In vitro release:}

The release of G-Rg3 from G-Rg3-PLP was investigated using the dialysis method. Briefly, volumes of G-Rg3LP and G-Rg3-PLP suspension equivalent to $2 \mathrm{mg}$ G-Rg3 were accurately measured and placed into dialysis bags. Then, the dialysis bags were immersed in $100 \mathrm{ml}$ dialysate $(30 \%$ ethanol PBS $(\mathrm{pH}=7.4))$ and the mixture was stirred at $120 \mathrm{rpm}$ at $37^{\circ}$. Samples ( $1 \mathrm{ml}$ of dialysate) were taken at predetermined times $(0,0.5,1,2,3,4,5,6,8,12,24,36,48,60$ and $72 \mathrm{~h})$, and replaced with an equal volume of fresh dialysate medium. The sample was filtered through a $0.45 \mu \mathrm{m}$ Nylon 66 membrane syringe filter and without any further treatment injected into HPLC-UV for G-Rg3 determination. The cumulative release rate was calculated according to the following formula, $\mathrm{F}(\%)=$ $\mathrm{Cn} \times \mathrm{V}+\sum_{\mathrm{n}=1}^{\mathrm{i}=\mathrm{n}=1} \mathrm{Ci} \times \mathrm{Vi} / \mathrm{Q} \times 100$, where $\mathrm{F}$ is the cumulative release rate, $\mathrm{Cn}$ is the dialysate drug concentration at a predetermined time, $\mathrm{V}$ is the total volume of dialysate, $\mathrm{Q}$ is the initial total drug dose before dialysis, $\mathrm{Ci}$ is the dialysate drug concentration at the last sample time, and $\mathrm{Vi}$ is the sample volume.

\section{Preparation of lyophilized G-Rg3-PLP:}

The G-Rg3-PLP suspension was mixed with different lyoprotectants and placed in a penicillin bottle for freeze-drying. The bottle was successively placed in
-20 and $-40^{\circ}$ refrigerators for prefreezing, followed by lyophilization using a freeze-dryer (BTP-3L-ES-55, SP Scientific, USA) at -52 to $-55^{\circ}$ for $48 \mathrm{~h}$. The lyophilized powder of G-Rg3-PLP was stored at $4^{\circ}$ for further use.

\section{RESULTS AND DISCUSSION}

G-Rg3-PLP was prepared using the film dispersionultrasonication method. As shown in fig. 1A, G-Rg3PLP exhibited spherical shape and were monodispersed. The particle size of G-Rg3-PLP was approximately $152.58 \pm 0.74 \mathrm{~nm}$ and the polydispersity index (PDI) was 0.293 (fig.1B), indicating that the particle size was uniform. The zeta potential was $-26.73 \pm 0.57 \mathrm{mV}$ (fig. 1C) as measured by a zeta potential analyser.

Liposomes are versatile drug carriers. Hydrophobic drugs are incorporated into the lipid bilayers, while hydrophilic drugs are usually encapsulated in the aqueous compartments. $\mathrm{EE}$ is one of the main evaluation indices for liposomes and was determined by comparing the concentration of $\mathrm{G}-\mathrm{Rg} 3$ in liposomes with the initial concentration of G-Rg3 (fig. 2). Therefore, the separation of free drug from liposomes is important. The determination method included highspeed centrifugation, dialysis, column chromatography, and microcolumn centrifugation. Considering the poor water solubility of G-Rg3, it is difficult to elute by column chromatography. Furthermore, the addition of organic solvent may lead to the leakage of liposomes. The dialysis method can be used to determine the EE of liposomes, because the dialysate is much larger than liposomes and free drug, it is easy to destroy the dynamic balance between them. Meanwhile, due to the poor stability of liposomes, the encapsulated drugs were prone to leakage. Sephadex chromatography requires a large sample loading quantity and has a long separation time. The process of eluent separation greatly dilutes the liposomes and free drug, which may cause liposomal leakage. Feix et al. proposed the microcolumn centrifugation method ${ }^{[25]}$, which has the advantage of only needing a small loading quantity of a sample, a small volume of eluent, and a short separation time.

In this study, both the microcolumn and high-speed centrifugation methods were investigated. For the microcolumn centrifugation method, Sephadex LH20 microcolumns were used, and the centrifugation speed was $1000 \mathrm{rpm}$. The microcolumn elution curve showed that it could separate the free drug and liposomes very well and that the average recovery was 97.19 $\pm 0.99 \%$, which indicated that this method was accurate 

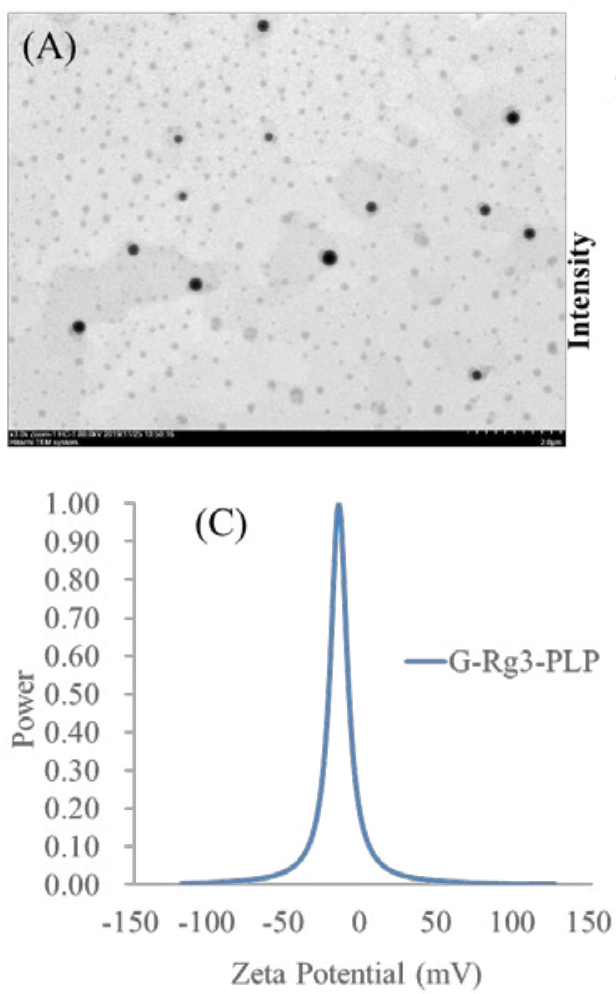

(B)



Fig. 1: The Characterization of G-Rg3-PLP

(A) TEM of G-Rg3-PLP, (B) Particle size of G-Rg3-PLP, (C) Zeta potential of G-Rg3-PLP ( - )

(A)

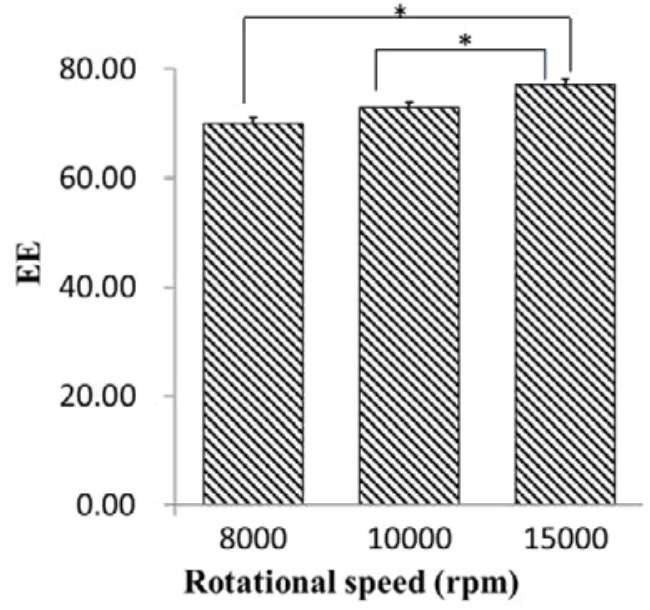

(B)

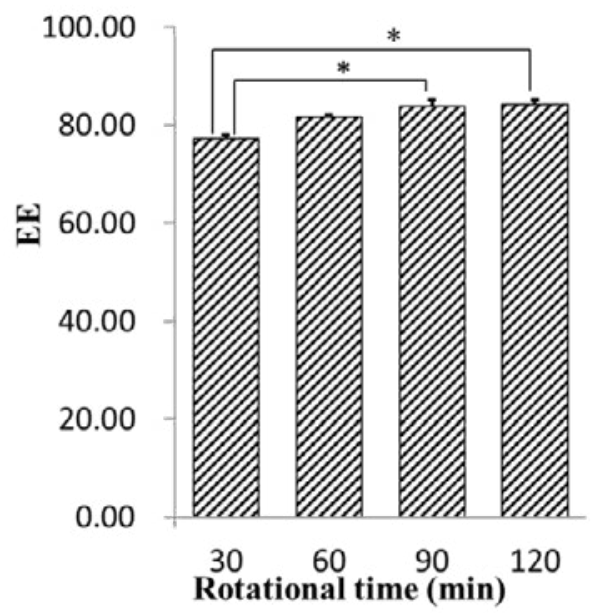

Fig. 2: The entrapment efficiency of G-Rg3

The influence of (A) different rotational speed or (B) different rotational time on The EE of G-Rg3, n=3, *p<0.05

and feasible. High-speed refrigerated centrifugation is a method used to separate free drug from liposomes by gravity. The rotational speed and rotational time were two factors influencing the accuracy of measurement results. The results showed that the EE of liposomes was significantly different at different centrifugal speeds. Increases in centrifugal speed led to an increase in the $\mathrm{EE}$ of liposomes. The highest EE was at $15000 \mathrm{rpm}$, which was similar to that measured by the microcolumn centrifugation method. When the centrifugation time was longer than $90 \mathrm{~min}$, the EE has no significant change. There was no significant difference in the $\mathrm{EE}$ of liposomes in response to different centrifugation times. EE increased slightly as centrifugation times increased. When the centrifugation time was more than 90 min, the EE did not change; thus, the optimal centrifugation speed and time were determined to be $15000 \mathrm{rpm}$ and $90 \mathrm{~min}$, respectively (the average recoveries of high, medium, and low concentration drug solutions was $98.27 \%$ ). Overall, both the Sephadex 
LH-20 microcolumn centrifugation and high-speed freezing centrifugation methods effectively separated G-Rg3-PLP and free G-Rg3. While the input sample quantity of high-speed freezing centrifugation is small, the operation is simple and can maintain the stability of liposomes in low temperatures. Therefore, the EE and DL of G-Rg3-PLP were measured using the highspeed freezing centrifugation method for the following experiments.

After determining the EE and DL determination method, the formulation of G-Rg3-PLP was investigated. According to the related references and data from preliminary experiments, the technological conditions (e.g., incubation temperature, hydration temperature) and the prescription parameters ( $\mathrm{pH}$ of $\mathrm{PBS}$ ) were screened by single factor investigation. The incubation temperature was set to $60^{\circ}$, the hydration temperature was $40^{\circ}$ and the $\mathrm{pH}$ of PBS was set to 7.4. Among these variables, the incubation temperature had a large impact on EE. This could be because as the acyl side in the lipid bilayer of liposomes changes from order to disorder, the film changes from gel crystalline to liquid crystalline at the phase-transition temperature ${ }^{[26]}$. This leads to a decrease in the thickness of the lipid bilayer and the cross-sectional area of the membrane and membrane fluidity increase ${ }^{[27]}$. When the incubation temperature was above the phase-transition temperature, the phospholipid membrane has greater fluidity and permeability and the drug loading increased ${ }^{[28]}$.

The amount of PC, Chol, and DSPE-PEG2000 in the liposomes was optimized by applying BBD. The corresponding values of BBD were observed and are shown in Table 1. Experimental data was fitted to a quadratic model by one-way analysis of variance (ANOVA). The results of the ANOVA for the 3 responses are shown in Table 2. The analysis showed that the models of DL and OD were significant at the $95 \%$ confidence level and that the lack of fit was not significant ( $p>0.05)$. Meanwhile, the model of EE was significant at the $95 \%$ confidence level and the lack of fit was significant $(p<0.05)$. Additionally, due to the complication and interaction of the response variables, a signal traditional forecasting model could not be relied to get accurate results for the EE and DL. Thus, the model of OD was chosen to analyse and predict the prescription and the results were considered adequate.

TABLE 1: BOX-BEHNKEN DESIGN AND THE CORRESPONDING RESPONSE MEASUREMENTS

\begin{tabular}{lcccccc}
\hline Run & $\mathrm{X}_{1}$ & $\mathrm{X}_{2}$ & $\mathrm{X}_{3}$ & $\mathrm{EE} \mathrm{( \% )}$ & $\mathrm{DL}(\%)$ & $\mathrm{OD}$ \\
\hline 1 & 1 & -1 & 0 & 81.32 & 6.14 & 0.163 \\
2 & -1 & 0 & -1 & 50.23 & 7.53 & 0 \\
3 & 0 & 0 & 0 & 78.66 & 6.64 & 0.313 \\
4 & -1 & -1 & 0 & 65.83 & 6.49 & 0 \\
5 & 0 & -1 & -1 & 84.93 & 7.06 & 0.514 \\
6 & 0 & 0 & 0 & 79.55 & 6.66 & 0.324 \\
7 & 0 & -1 & 1 & 66.26 & 5.11 & 0 \\
8 & -1 & 0 & 1 & 39.38 & 4.95 & 0 \\
9 & -1 & 1 & 0 & 67.47 & 6.75 & 0 \\
10 & 0 & 0 & 0 & 84.21 & 7.32 & 0.559 \\
11 & 1 & 1 & 0 & 85.48 & 7.01 & 0.510 \\
12 & 1 & 0 & 1 & 74.24 & 5.31 & 0 \\
13 & 0 & 0 & 0 & 83.33 & 6.74 & 0.405 \\
14 & 0 & 0 & 0 & 82.15 & 6.69 & 0.374 \\
15 & 1 & 0 & -1 & 80.01 & 7.21 & 0.449 \\
16 & 0 & 1 & 1 & 73.22 & 5.16 & 0 \\
17 & 0 & 1 & -1 & 86.37 & 7.43 & 0.625 \\
\hline$X_{1}$ is & & &
\end{tabular}

$X_{1}$ is PC: Chol ratio, $X_{2}$ is PC:DSPE- PEG $_{2000}$ ratio, $X_{3}$ is PC: $G-R g 3$ ratio, $E E$ is encapsulation efficiency, $D L$ is drug-loading rate, $O D$ is overall desirability

TABLE 2: ANOVA FOR RESPONSE SURFACE QUADRATIC OF OD

\begin{tabular}{|c|c|c|c|c|c|c|}
\hline Source & Sum of Squares & df & Mean Squares & $F$ value & $P$ value & \\
\hline Model & 0.79 & 9 & 0.088 & 5.52 & 0.0173 & Significant \\
\hline$X_{1}$ & 0.16 & 1 & 0.16 & 9.84 & 0.0165 & \\
\hline$x_{2}$ & 0.026 & 1 & 0.026 & 1.64 & 0.2412 & \\
\hline$x_{3}$ & 0.32 & 1 & 0.32 & 19.71 & 0.0030 & \\
\hline$x_{1} x_{2}$ & 0.030 & 1 & 0.030 & 1.88 & 0.2124 & \\
\hline$X_{1} X_{3}$ & 0.050 & 1 & 0.050 & 3.15 & 0.1191 & \\
\hline$x_{2} x_{3}$ & $3.080 \mathrm{E}-003$ & 1 & $3.080 \mathrm{E}-003$ & 0.19 & 0.6740 & \\
\hline$X_{12}$ & 0.17 & 1 & 0.17 & 10.49 & 0.0143 & \\
\hline$x_{22}$ & $3.098 \mathrm{E}-003$ & 1 & $3.098 \mathrm{E}-003$ & 0.19 & 0.6731 & \\
\hline$x_{32}$ & 0.029 & 1 & 0.029 & 1.82 & 0.2194 & \\
\hline Residual & 0.11 & 7 & 0.016 & & & \\
\hline Lack of Fit & 0.073 & 3 & 0.024 & 2.47 & 0.2010 & not significant \\
\hline Pure Error & 0.039 & 4 & $9.801 \mathrm{E}-003$ & & & \\
\hline Cor Total & 0.91 & 16 & & & & \\
\hline
\end{tabular}


The ANOVA test for OD indicated that the linear model was significant $(\mathrm{p}<0.05)$. The model of OD was fitted by Design-Expert 8.0.6 software and a formula was established. The resulting regressive equation, which rejects the undistinguished variables with the F-test of every index in terms of coded values was as follows: $\mathrm{OD}=-1.38399+0.19428 \mathrm{X}_{1}+0.16665 \mathrm{X}_{3}-6.59917 \mathrm{E}-$ $003 X_{1}^{2}\left(R^{2}=0.8766\right)$.

The result of the regression OD equation indicated that $\mathrm{R}^{2}=0.8766$, meaning it is suitable for the desired model. The 3D surface model of the 3 indices of EE, DL and OD with independent variables is shown in fig. 3. The software predicted the optimum PC:Chol, PC:DSPE$\mathrm{PEG}_{2000}$ and PC:G-Rg3 ratios, which were 14:1, $5 \%$ and $8: 1$, respectively. The predicted entrapment efficiency and drug loading rates were 87.71 and $7.50 \%$, respectively. A verification experiment was completed, and the result was consistent with theoretical predictions.

In this study, the cumulative release rate of G-Rg3 from G-Rg3-PLP or G-Rg3-LP was investigated. Rg3 has low solubility in water, which means a delivery vehicle is needed if this drug is to be administered systemically ${ }^{[6]}$. Therefore, in this study, the release profiles of G-Rg3LP and G-Rg3-PLP were investigated. Due to the low water solubility of G-Rg3, $30 \%$ ethanol PBS $(\mathrm{pH}=7.4)$ was selected as the dialysate to improve the solubility of G-Rg3; the results are shown in fig. 4. Compared with G-Rg3-LP, G-Rg3-PLP showed a more obvious sustained release effect. This may be due to the steric effect of the polyethylene glycol (PEG) chain, which can prevent drug diffusion ${ }^{[28]}$. Both groups did not display a burst effect at their initial release patterns.

Considering that aggregation, fusion, precipitation, and drug leakage may occur during storage and transportation $^{[10]}$, the stability of G-Rg3-PLP needs to be improved. Freeze-drying is a promising method to extend the life shelf of liposomes; however, the sublimation of ice or crystallization may occur during the phase transition, which can disrupt the integrity of lipid membranes. Therefore, the addition of lyoprotectants is necessary to prevent changes in appearance, increases in particle size, and decreases in drug loading ${ }^{[29,30]}$. Different lyoprotectants were screened in this study according to the appearance, re-dispersity, particle size, and entrapment efficiency of lyophilization of G-Rg3PLP (Table 3). When mannitol and PEG6000 are used as freeze-drying protectors, the redispersion effect is poor and the particle size is large. When hydroxypropyl$\beta$-cyclodextrin is used as freeze-drying protectant, the encapsulation efficiency changes greatly. When glucose is used as freeze-drying protective agent, the appearance of the freeze-dried product is poor. Finally, two percent lactose was chosen as the protectant because its reconstruction time is short, the particle size is small, and the encapsulation efficiency changed only slightly.
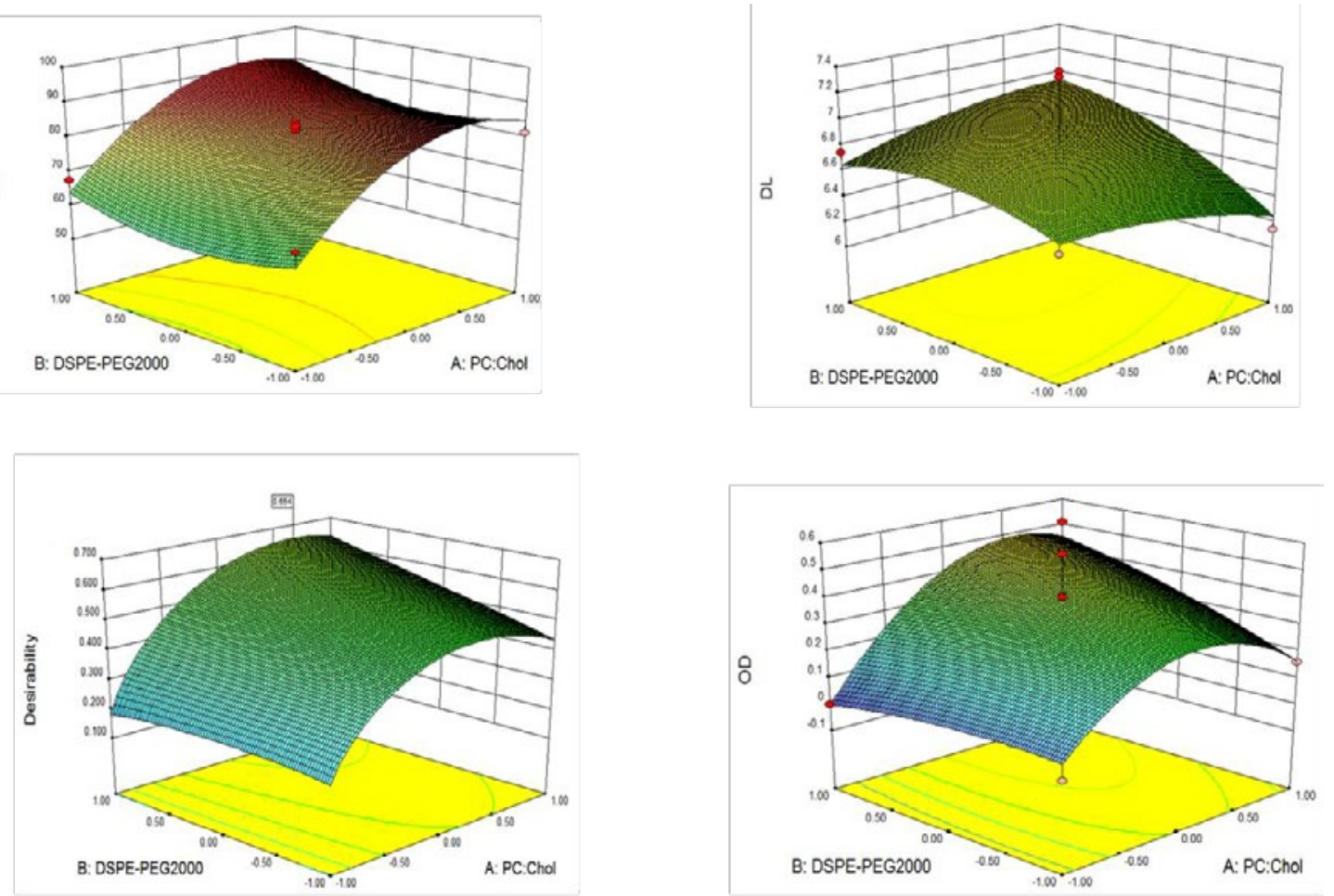

Fig. 3: Response 3D plots

A. EE ; B. DL ; C. OD ; D. desirability 
TABLE 3: SCREENING RESULTS OF DIFFRERNT LYOPROTECTANTS

\begin{tabular}{lccccc}
\hline lyoprotectant & percent & Appearance & $\mathrm{EE}(\%)$ & $\mathrm{D}_{50}(\mathrm{~nm})$ & Reconstruction time $(\mathrm{s})$ \\
\hline \multirow{3}{*}{ Glucose } & 2 & + & $76.04 \pm 4.06$ & $74.1 \pm 9.78$ & $7.33 \pm 0.58$ \\
& 4 & + & $69.99 \pm 5.55$ & $79.1 \pm 14.92$ & $8.33 \pm 2.08$ \\
Lactose & 6 & + & $68.64 \pm 10.62$ & $102.7 \pm 3.19$ & $6.33 \pm 1.15$ \\
& 2 & ++ & $78.15 \pm 1.90$ & $105.3 \pm 11.41$ & $9.33 \pm 0.58$ \\
& 4 & ++ & $71.60 \pm 7.48$ & $79.8 \pm 9.96$ & $9.00 \pm 3.61$ \\
Mannitol & 6 & ++ & $71.39 \pm 5.04$ & $64.0 \pm 3.37$ & $6.00 \pm 2.00$ \\
& 2 & +++ & $78.46 \pm 3.37$ & $175.7 \pm 11.06$ & $11.33 \pm 2.89$ \\
& 4 & +++ & $77.69 \pm 1.62$ & $174.9 \pm 7.61$ & $9.66 \pm 3.06$ \\
PEG & 6 & +++ & $76.17 \pm 1.62$ & $186.5 \pm 3.34$ & $10.67 \pm 0.58$ \\
& 2 & +++ & $81.69 \pm 4.40$ & $186.9 \pm 8.30$ & $18.00 \pm 2.65$ \\
& 4 & +++ & $81.01 \pm 3.67$ & $175.6 \pm 9.30$ & $11.67 \pm 1.53$ \\
HP-B-CD & 6 & +++ & $79.89 \pm 2.42$ & $209.8 \pm 12.32$ & $9.67 \pm 0.58$ \\
& 2 & ++ & $67.89 \pm 13.36$ & $63.0 \pm 2.05$ & $14.00 \pm 2.00$ \\
& 4 & ++ & $62.63 \pm 5.62$ & $64.3 \pm 7.49$ & $12.67 \pm 2.31$ \\
& 6 & ++ & $53.56 \pm 11.60$ & $65.0 \pm 2.15$ & $14.67 \pm 3.79$ \\
\hline
\end{tabular}

$\mathrm{PEG}_{6000}$ is poly (ethylene glycol) $6000, \mathrm{HP}-\mathrm{B}-\mathrm{CD}$ is hydroxypropyl-B-cyclodextrin, EE is encapsulation efficiency, $\mathrm{n}=3, \mathrm{mean} \pm \mathrm{SD}$, appearance : white, atrophy, surface irregularities, roughness, “+” ; white, a little atrophy, surface irregularities slightly, a little smooth, “++" ; white, satiation, surface neatness, smooth, “+++"

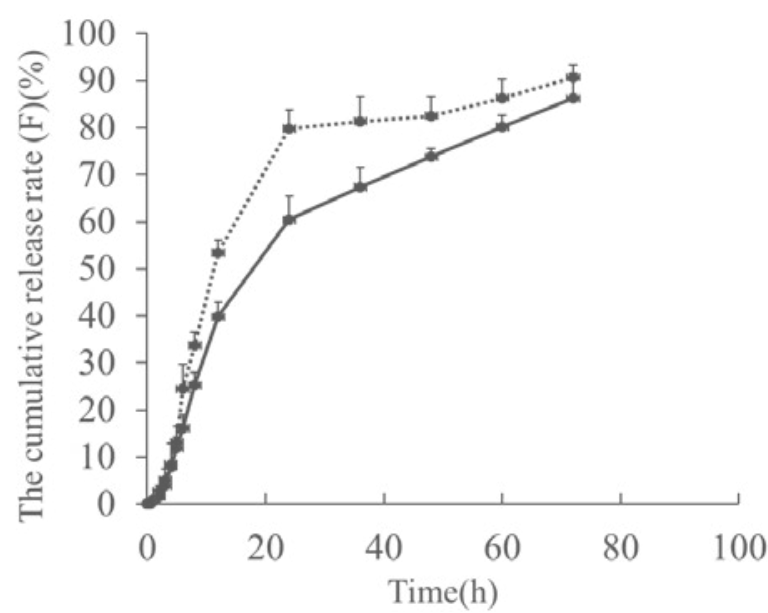

Fig. 4: The cumulative release rate (F) of G-Rg3-LP and G-Rg3PLP

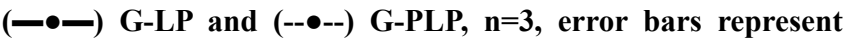
standard deviation for $n=3$

The protection mechanism of lyophilized protectants can be divided into the water replacement hypothesis and the vitrification model. The water replacement hypothesis was first proposed by Crowe et al..$^{[31]}$, in which sugars reduce the interactions between the water and phospholipids and eventually replace the water. In this process, the head group spacing is maintained and van der Waals interactions among the acyl chains of phospholipids are reduced ${ }^{[32,33]}$. The other lyoprotective mechanism is the vitrification model proposed by Koster etal. ${ }^{[34]}$ The sugar solution generates a stable glassy state during freezing at low temperature, reduces crystallization during lyophilization, effectively prevents the aggregation of polymer particles, and protects the integrity of the structure of the bilayer of liposome phospholipids ${ }^{[35]}$.

In this study, G-Rg3-PLP was successfully prepared using film dispersion-ultrasonic technology. G-Rg3PLP had a small particle size and high EE. In vitro release showed that G-Rg3-PLP had a more obvious sustained release effect, which suggests that G-Rg3PLP may enhance the therapeutic effect of G-Rg3. The future work should focus on the pharmacokinetics of G-Rg3-PLP and its anticancer therapeutic effect.

\section{Acknowledgements:}

This work was supported by grants from the National Natural Science Foundation of China (Grant No. 81560578 and 81860304) and Natural Science Research Foundation of the Education Department of Jilin Province (Grant No. JJKH20191158KJ).

\section{DISCLOSURE}

All authors declare no financial competing interests.

\section{REFERENCES}

1. Pan Y, Guo H, Han J. Ginsenoside Rg3 attenuates cell migration via inhibition of aquaporin 1 expression in PC-3M prostate cancer cells. Eur J Pharmacol 2012;683(1-3):27-34.

2. Zheng JC. Pharmaceutical Botany. Beijing, China: People's Medical Publishing House 1999. p. 217.

3. Lee YJ, Lee S, Ho JN. Synergistic antitumor effect of ginsenoside $\mathrm{Rg} 3$ and cisplatin in cisplatin-resistant bladder tumor cell line. Oncol Rep 2014;32(5):1803-8.

4. Kim SM, Lee SY, Chol JS. Combination of ginsenoside Rg3 with docetaxel enhances the susceptibility of prostate 
cancer cells via inhibition of NF-kappaB. Euro J Pharmacol 2010;631(1-3):1-9.

5. Zhang Q, Kang X, Yang B. Antiangiogenic effect of capecitabine combined with ginsenoside Rg3 on breast cancer in mice. Cancer Biother Radiopharm 2008;23(5)647-53.

6. Yu H, Teng L, Meng Q. Development of liposomal ginsenoside Rg3: Formulation optimization and evaluation of its anticancer effects. Int J Pharm 2013;450(1-2):250-8.

7. Ye FF, Dai Y, Xu H. Preparation and characterization of ginsenosides $\mathrm{Rg} 3$ liposomes and their inhibitory effect on melanoa cells. Chin J New Drugs 2014;23(21):2542-6.

8. Zhang MM. The preparation of inclusion complex of 20(S)protopanoxadiol-hydroxypropyl- $\beta$-cyclodextrin [dissertation]. Yangzhou, China: Yangzhou University; 2016.

9. Geng L, Fan J, Gao QL. Preliminary study for the roles and mechanisms of 20 (R) -ginsenoside Rg3 and PEG-PLGA$\mathrm{Rg} 3$ nanoparticles in the Lewis lung cancer mice. Beijing Da Xue Xue Bao Yi Xue Ban 2016;48(3):496-501.

10. Allen M, Cullis R. Liposomal drug delivery systems: From concept to clinical applications. Adv Drug Deliv Rev 2013;65(1):36-48.

11. Blume G, Cevc G. Molecular mechanism of the lipid vesicle longevity in vivo. Biochimica et Biophysica Acta 1993;1146(2):157-68.

12. Gabizon A. Pegylated Liposomal Doxorubicin: Metamorphosis of an Old Drug into a New Form of Chemotherapy. Cancer Invest 2001;19(4):424-36.

13. Yang S. Preparation, in vitro Characterization and Pharmacokinetic Study of Coenzyme Q10 Long-Circulating Liposomes. Drug Res 2018;68(5):270-9.

14. Zhao T, Liu Y, Gao Z. Self-assembly and cytotoxicity study of PEG-modified ursolic acid liposomes. Mater Sci Eng C Mater Biol Appl 2015;53:196-203.

15. Luo D, Carter A, Raz A. Doxorubicin encapsulated in stealth liposomes conferred with light-triggered drug release. Biomaterials 2016;75:193-202.

16. Abdelbary A, AbouGhaly H. Design and optimization of topical methotrexate loaded niosomes for enhanced management of psoriasis: Application of Box-Behnken design, in vitro evaluation and in vivo skin deposition study. Int $\mathrm{J}$ Pharm 2015;485(1-2):235-43.

17. Response surface methodology. Wikipedia, The Free Encyclopedia; 2019 Feb 9, 14:07 UTC [cited 2019 Apr 3]. Available from: https://en.wikipedia. org/w/index.php?tit le=Response_surface methodology\&oldid $=882493447$.

18. Kant R, Bodla B, Bhutani R. Enantioselective Box Behenken Optimized HPLC-DAD Method for the Simultaneous Estimation of Alogliptin Enantiomorphs in Pharmaceutical Formulations and their Pharmacokinetic Study in Rat Plasma. Adv Pharm Bull 2019;9(1):147-58.

19. Jin $\mathrm{H}, \mathrm{Lu} \mathrm{Q}$, Jiang G. Curcumin liposomes prepared with milk fat globule membrane phospholipids and soybean lecithin. J Dairy Sci 2016;99(3):1780-90.
20. Sun J, Deng Y, Wang S. Liposomes incorporating sodium deoxycholate for hexamethylmelamine (HMM) oral delivery: Development, characterization, and in vivo evaluation. Drug Deliv 2010;17(3):164-70.

21. Zhu J, Zhang W, Wang D. Preparation and characterization of norcantharidin liposomes modified with stearyl glycyrrhetinate. Exp Ther Med 2018;16(3)1639-46.

22. Cheung CCL, Al-Jamal WT. Sterically stabilized liposomes production using staggered herringbone micromixer: Effect of lipid composition and PEG-lipid content. Int $\mathrm{J}$ Pharm 2019;566:687-96.

23. Paolino D, Accolla ML, Cilurzo F. Interaction between PEG lipid and DSPE/DSPC phospholipids: An insight of PEGylation degree and kinetics of de-PEGylation. Colloids Surf B Biointerfaces 2017;155:266-75.

24. Yan $\mathrm{H}$, Wei P, Song J. Enhanced anticancer activity in vitro and in vivo of luteolin incorporated into long-circulating micelles based on DSPE-PEG2000 and TPGS. J Pharm Pharmacol 2016;68(10):1290-8.

25. Feix JB. Weissing V. Liposomes: A practical Approach Second Edition. Quarterly Review of Biology. Oxford, United Kingdom: Oxford University Press; 2004. p. 264.

26. Kannan V, Balabathula P, Divi K. Optimization of drug loading to improve physical stability of paclitaxel-loaded longcirculating liposomes. J Liposome Res 2015;25(4):308-15.

27. Hermetter A, Kopec W, Khandelia H. Conformations of double-headed, triple-tailed phospholipid oxidation lipid products in model membranes. Biochim Biophys Acta 2013;1828(8):1700-6.

28. Chen J, Cheng D, Li J. Influence of lipid composition on the phase transition temperature of liposomes composed of both DPPC and HSPC. Drug Dev Ind Pharm 2013;39(2):197-204.

29. Yu G, Okano T, Kataoka K. Polymeric micelles for drug delivery: Solubilization and haemolytic activity of amphotericin B. J Control Release 1998;53(1-3):131-6.

30. $\mathrm{Li} \mathrm{J}, \mathrm{Hu} \mathrm{M}, \mathrm{Xu} \mathrm{H}$. Influence of type and proportion of lyoprotectants on lyophilized ginsenoside Rg3 liposomes. J Pharm Pharmacol 2016;68(1):1-13.

31. Crowe LM, Womersley C, Crowe JH. Prevention of fusion and leakage in freeze-dried liposomes by carbohydrates. Biochim Biophys Acta 1986;861:131-40.

32. Crowe JH, Hoekstra FA, Nguyen KH. Is vitrification involved in depression of the phase transition temperature in dry phospholipids? Biochim Biophys Acta 1996;1280:187-96.

33. Crowe LM, Crowe JH. Trehalose and dry dipalmitoylphosphatidylcholine revisited. Biochim Biophys Acta 1988;964:193-201.

34. Koster KL, Webb MS, Bryant G. Interactions between soluble sugars and POPC (1-palmitoyl-2-oleoylphosphatidylcholine) during dehydration: vitrification of sugars alters the phase behavior of the phospholipids. Biochim Biophys Acta 1984;1193:143-50.

35. Sun WQ, Leopold AC, Crowe LM. Stability of dry liposomes in sugar glasses. Biophysical Journal 1996;70(4):1769-76. 